\title{
APPROXIMATION BY A POLYNOMIAL AND ITS DERIVATIVES ON CERTAIN CLOSED SETS
}

\author{
ERRETT BISHOP
}

The work on the theory of approximations initiated by Weierstrass and continued by Walsh, Keldysh, and Lavrentiev, among others, has culminated in the following theorem of Mergelyan (See Mergelyan [3]): Given any compact subset $C$ of the complex plane, which does not separate the plane, and given any continuous function $f$ on $C$ which is analytic interior to $C$, then $f$ can be approximated uniformly on $C$ by polynomials.

This theorem leaves the following question unanswered: If $f_{0}, f_{1}, \cdots, f_{n}$ are continuous functions on $C$, can a sequence $\left\{p_{k}\right\}$ of polynomials be found with the property that for each integer $i$ with $0 \leqq i \leqq n$ the sequence $\left\{p_{\boldsymbol{k}}^{[i]}\right\}$, where $p_{\boldsymbol{k}}^{[i]}$ denotes the $i$ th derivative of $p_{k}$, converges uniformly on $C$ to $f_{i}$ ? If $C$ is totally disconnected, it is easy to show that the answer to this question is always yes. We omit the simple proof, because a more general result will be given elsewhere. If $C$ is a Jordan arc, the question becomes more complicated. It is clear that if $C$ has a rectifiable sub-arc $J$, whose endpoints we call $z_{0}$ and $z_{1}$, then for the approximation to be possible it is necessary that $\int_{J} f_{i+1}(z) d z=f_{i}\left(z_{1}\right)-f_{i}\left(z_{0}\right)$ for $0 \leqq i \leqq n-1$. Thus, if the approximation is to be possible whatever the functions $f_{0}, f_{1}, \cdots, f_{n}$, it is necessary that $C$ have no rectifiable sub-arcs. Conversely, if $C$ is a Jordan arc having no rectifiable sub-arcs, we conjecture that the approximation is always possible. It is the purpose of this paper to prove this conjecture by means of an additional hypothesis, that $C$ satisfy a Lipschitz condition of a fixed order $c$ at a dense set of points. (This concept will be defined below.) The author has been unable to prove the conjecture without this restriction.

If $S_{1}$ and $S_{2}$ are any subsets of the complex plane, define $d\left(S_{1}, S_{2}\right)$ $=\min \left\{\left|z_{1}-z_{2}\right| \mid z_{1} \in S_{1}, z_{2} \in S_{2}\right\}$.

Definition 1 . Let $\phi$ be a homeomorphic map of $[0,1]$ into the complex plane, so that $\phi[0,1]$ is a Jordan arc $C$. We say that $C$ satisfies a Lipschitz condition of order $c$ at a point $\phi(t)$ of $C, t \in[0,1]$, if there exist $A>0$ and $\delta>0$ such that $\max \{d(\phi[0, t], z), d(\phi[t, 1], z)\}$ $\geqq A|\phi(t)-z|^{c}$ whenever $|\phi(t)-z|<\delta$.

Then we have

THEOREM 1. If $C$ has no rectifiable sub-arcs and if there exists $c>0$ such that $C$ satisfies a Lipschitz condition of order $c$ at a dense set $S$ of

Received by the editors April 23, 1957, and, in revised form, May 3, 1958. 
points, then for any continuous functions $f_{0}, \cdots, f_{n}$ on $C$ there exists a sequence $\left\{p_{i}\right\}$ of polynomials for which $p_{i}^{[k]} \rightarrow f_{k}$ uniformly on $C$ as $i \rightarrow \infty$, for $0 \leqq k \leqq n$.

The proof of Theorem 1 will utilize the Riesz representation theorem (see Banach $\left[1\right.$, p. 60]). If $X$ is the set of all $n+1$ tuples $\left(f_{0}, \cdots\right.$, $f_{n}$ ) of continuous functions on $C$, topologized by the norm $\|\left(f_{0}, \cdots\right.$, $\left.f_{n}\right) \|=\sup \left\{\left|f_{i}(z)\right| \mid z \in C, 0 \leqq i \leqq n\right\}$, then by the Riesz theorem we see that to any bounded linear functional $L$ on $X$ correspond unique measures $\mu_{0}, \cdots, \mu_{n}$ on $[0,1]$ such that $L\left(f_{0}, \cdots, f_{n}\right)$ $=\int_{0}^{1} f_{0}(\phi(t)) d \mu_{0}(t)+\cdots+\int_{0}^{1} f_{n}(\phi(t)) d \mu_{n}(t)$. Now let $Y$ be the subset of $X$ consisting of all $\left(p, p^{[1]}, \cdots, p^{[n]}\right)$, where $p$ is any polynomial. Then Theorem 1 states that $Y$ is dense in $X$. By the Hahn-Banach theorem (see Banach [1]), this is equivalent to saying that every bounded linear functional on $X$ which vanishes on $Y$ vanishes on $X$. If $L$ is the bounded linear functional in question, then by the above representation of $L$ we see that $L\left(p, \cdots, p^{[n]}\right)=\int_{0}^{1} p(\phi(t)) d \mu_{0}(t)$ $+\cdots+\int_{0}^{1} p^{[n]}(\phi(t)) d \mu_{n}(t)=0$ for all polynomials $p$. To prove Theorem 1 we must show that this implies that $\mu_{0}=\mu_{1}=\cdots=\mu_{n}=0$. The linear functional $L$ and therefore $\mu_{0}, \cdots, \mu_{n}$ will be fixed during the discussion. As an abbreviation we set $L_{t}(p)=\int_{0}^{t} p(\phi(t)) d \mu_{0}(t)$ $+\cdots+\int_{0}^{t} p^{[n]}(\phi(t)) d \mu_{n}(t)$ for all $t$ in $[0,1]$ and all functions $p$ analytic in some neighborhood of $C$. Then $L_{1}(p)=0$.

Assume now that $\phi(t) \in S$. We proceed to obtain a new formula for $L_{t}(p)$. To do this, take $A$ and $\delta$ as in Definition 1, and let $\epsilon$ be positive and less than $\delta$. Let $J$ be the circle $|z-\phi(t)|=\epsilon$. Then by Definition 1 , for $z$ in $J$ either $d\left(z, U_{1}\right)>A \epsilon^{c}$ or $d\left(z, U_{2}\right)>A \epsilon^{c}$, where $U_{1}=\phi[0, t]$ and $U_{2}=\phi[t, 1]$. Thus we can write $J=J_{1} \cup J_{2}$, where $J_{1}$ and $J_{2}$ are disjoint Borel sets and $d\left(z, U_{i}\right)>A \epsilon^{c}$ for $z$ in $J_{i}$. Now if $p$ is any polynomial, let $K=\max \{|p(z)| \mid z \in J\}$. Then

$$
p(z)=(1 / 2 \pi i) \int_{J} p(\zeta) d \zeta /(\zeta-z)
$$

for $|z-\phi(t)|<\epsilon$. If for $i=1$ and 2 we define

$$
f_{i}(z)=(1 / 2 \pi i) \int_{J_{i}} p(\zeta) d \zeta /(\zeta-z),
$$

then we see that $f_{i}$ is analytic on the complement of the closure of $J_{i}$, that $\left|f_{i}^{[j]}(z)\right| \leqq K j ! d\left(z, J_{i}\right)^{-(j+1)}$, and that $p(z)=f_{1}(z)+f_{2}(z)$ for $|z-\phi(t)|<\epsilon$. Thus we see that $\left|f_{i}^{[j]}(z)\right|<K j !\left[A \epsilon^{c}\right]^{-(j+1)}$ for $z$ in $U_{i}$.

Now let $0_{1}$ be a neighborhood of $\phi[0, t]$ and $0_{2}$ a neighborhood of $\phi[t, 1]$ such that $0_{1} \cap 0_{2}=\{z|| z-\phi(t) \mid<\epsilon\}$ and $0_{1} \cap J_{1}=0_{2} \cap J_{2}=\phi$. Then $f_{1}+f_{2}=p$ on $0_{1} \cap 0_{2}$ and $f_{i}$ is analytic in $0_{i}$. Therefore we may 
define an analytic function $g_{1}$ on $0_{1} \cup 0_{2}$ by specifying $g_{1}(z)=f_{1}(z)$ for $z$ in $0_{1}$ and $g_{1}(z)=p(z)-f_{2}(z)$ for $z$ in $0_{2}$. Also define $g_{2}$ on $0_{1} \cup 0_{2}$ by $g_{2}(z)=f_{2}(z)$ for $z$ in $0_{2}$ and $g_{2}(z)=p(z)-f_{1}(z)$ for $z$ in $0_{1}$. Then $g_{1}+g_{2}=p$ in $0_{1} \cup 0_{2}$. Also $\left|g_{i}^{[j]}(z)\right|=\left|f_{i}^{[j]}(z)\right| \leqq K j !\left[A \epsilon^{c}\right]^{-(j+1)}$ for $z$ in $U_{i}$, where $i=1$ or 2 and $j$ is arbitrary. For $i=1$ this inequality, in conjunction with the definition of $L_{t}\left(g_{1}\right)$, tells us that $\left|L_{t}\left(g_{1}\right)\right| \leqq M_{1} K \epsilon^{-c(n+1)}$, where $M_{1}$ is a constant. For $i=2$ the inequality tells us that $\mid L\left(g_{2}\right)$ $-L_{t}\left(g_{2}\right) \mid \leqq M_{2} K \epsilon^{-c(n+1)}$. Thus we see that $\left|L_{t}(p)\right|=\left|L_{t}\left(g_{1}\right)+L_{t}\left(g_{2}\right)\right|$ $\leqq\left|L_{t}\left(g_{1}\right)\right|+\left|L_{t}\left(g_{2}\right)\right|=\left|L_{t}\left(g_{1}\right)\right|+\left|L_{t}\left(g_{2}\right)-L\left(g_{2}\right)\right| \leqq M K \epsilon^{-m}$, where $M=M_{1}+M_{2}$ and $m$ is any integer larger than $c(n+1)$. Taking $p$ to be the polynomial $(z-\phi(t))^{i}$, we see that $K=\epsilon^{j}$, so that $L_{t}\left([z-\phi(t)]^{j}\right)$ $<M \epsilon^{j-m}$ for all $\epsilon<\delta$. If $j>m$, this implies that $L_{t}\left([z-\phi(t)]^{j}\right)=0$. Therefore $L_{t}(p)$ depends only on the first $m+1$ terms of the expansion of $p$ in powers on $z-\phi(t)$, so that

$$
L_{t}(p)=\sum_{i=0}^{m} \beta_{i}(t) p^{[i]}(\phi(t)), \quad \text { where } \quad \beta_{0}(t), \cdots, \beta_{m}(t)
$$

are certain complex numbers.

For the remainder of the proof, the only use which will be made of the fact that $C$ satisfies a Lipschitz condition of order $c$ at points of $S$ will be to conclude that the expression just obtained for $L_{t}(p)$ is valid whenever $\phi(t)$ is in $S$. Therefore, the conjecture of the introductory paragraphs can be proved whenever the expression just obtained for $L_{t}(p)$ can be shown to be valid for a set of values of $t$ which is dense in $[0,1]$.

We now obtain another formula for $L_{t}(p)$, where now $t$ may be any point in $(0,1)$. For any polynomial $p$ and any complex number $z$ we have the Taylor's formula

$$
p(z)=\sum_{i=0}^{\infty} p^{[i]}(\phi(t)) \frac{[z-\phi(t)]^{i}}{i !} .
$$

Thus

$$
\begin{aligned}
L_{t}(p) & =\sum_{j=0}^{n} \int_{0}^{t}\left\{\sum_{i=0}^{\infty} p^{[i+j]}(\phi(t)) \frac{[\phi(x)-\phi(t)]^{i}}{i !}\right\} d \mu_{j}(x) \\
& =\sum_{i=0}^{\infty} p^{[i]}(\phi(t))\left(\sum_{j=0}^{i} \int_{0}^{t} \frac{[\phi(x)-\phi(t)]^{i-j}}{(i-j) !} d \mu_{j}(x)\right)
\end{aligned}
$$

where $\mu_{j}=0$ if $j>n$. If we define

$$
\alpha_{i}(t)=\sum_{j=0}^{i} \int_{0}^{t} \frac{[\phi(x)-\phi(t)]^{i-j}}{(i-j) !} d \mu_{j}(x),
$$


for $t$ in $(0,1)$, we therefore have $L_{t}(p)=\sum_{i=0}^{\infty} p^{[i]}(\phi(t)) \alpha_{i}(t)$. We see that $\alpha_{i}$ is continuous on the right. Comparing the two formulas obtained for $L_{t}(p)$, we see that $\sum_{i=0}^{m} \beta_{i}(t) p^{[i]}(\phi(t))=\sum_{i=0}^{\infty} \alpha_{i}(t) p^{[i]}(\phi(t))$ for all $t$ in $(0,1)$ with $\phi(t) \in S$, and for all polynomials $p$. It follows that $\alpha_{i}(t)=0$ for $i>m, t \in(0,1)$, and $\phi(t) \in S$. Since $S$ is dense and since $\alpha_{i}$ is continuous on the right, it follows that $\alpha_{i}=0$ for $i>m$. Before proceeding, we need a definition.

Definition 2. If $f$ and $g$ are two complex valued functions on $[0,1]$, if $t \in[0,1)$, and if $a$ is a complex number, then $a$ is said to be a right conditional derivative at the point $t$ of $f$ with respect to $g$ if there exists a sequence $\left\{t_{i}\right\}$, with $t_{i} \in(t, 1), t_{i} \rightarrow t$ as $i \rightarrow \infty$, and

$$
\left[f\left(t_{i}\right)-f(t)\right]\left[g\left(t_{i}\right)-g(t)\right]^{-1} \rightarrow a \text { as } i \rightarrow \infty .
$$

To get more information about $\alpha_{i}$, take $0<t_{0}<t<1$, set $\lambda_{i}(t)$ $=\mu_{i}[0, t]=\int_{0}^{t} d \mu_{i}(t)$, and consider the difference quotient

$$
\begin{aligned}
& \left\{\left[\alpha_{i}(t)-\lambda_{i}(t)\right]-\left[\alpha_{i}\left(t_{0}\right)-\lambda_{i}\left(t_{0}\right)\right]\right\}\left[\phi(t)-\phi\left(t_{0}\right)\right]^{-1} \\
& =\left\{\sum_{j=0}^{i-1} \int_{0}^{t} \frac{[\phi(x)-\phi(t)]^{i-j}}{(i-j) !} d \mu_{j}(x)\right. \\
& \left.\quad-\sum_{j=0}^{i-1} \int_{0}^{t_{0}} \frac{\left[\phi(x)-\phi\left(t_{0}\right)\right]^{i-j}}{(i-j) !} d \mu_{j}(x)\right\}\left[\phi(t)-\phi\left(t_{0}\right)\right]^{-1} \\
& =\sum_{j=0}^{i-1} \int_{0}^{t_{0}} \frac{[\phi(x)-\phi(t)]^{i-j}-\left[\phi(x)-\phi\left(t_{0}\right)\right]^{i-j}}{(i-j) !}\left[\phi(t)-\phi\left(t_{0}\right)\right]^{-1} d \mu_{j}(x) \\
& \quad+\sum_{j=0}^{i-1} \int_{t_{0}<x \leqq t} \frac{[\phi(x)-\phi(t)]^{i-j}}{(i-j) !}\left[\phi(t)-\phi\left(t_{0}\right)\right]^{-1} d \mu_{j}(x) .
\end{aligned}
$$

The second of these summations less than

$$
\sum_{j=0}^{i-1} \int_{t_{0}<x \leqq t} \frac{2 \cdot(2 B)^{i-j-1}}{(i-j) !}\left|d \mu_{j}(x)\right|
$$

in absolute value, if $B$ is the bound of $|\phi(x)|$ and if $t$ is chosen so that $\left|\phi(t)-\phi\left(t_{0}\right)\right| \geqq 2^{-1}|\phi(x)-\phi(t)|$ for all $x$ in $\left[t_{0}, t\right]$. The latter condition will be satisfied if $\left|\phi(t)-\phi\left(t_{0}\right)\right| \geqq\left|\phi(x)-\phi\left(t_{0}\right)\right|$ for all $x$ in $\left[t_{0}, t\right]$, and values of $t$ can be found arbitrarily close to $t_{0}$ for which this will be true. Thus the second summation can be made arbitrarily small for certain values of $t$ close to $t_{0}$. Due to uniform convergence under the integral signs, the other of the above summations approaches

$$
\sum_{j=0}^{i-1}-\int_{0}^{t_{0}} \frac{\left[\phi(x)-\phi\left(t_{0}\right)\right]^{i-j-1}}{(i-j-1) !} d \mu_{j}(x)=-\alpha_{i-1}\left(t_{0}\right) \text { as } t \rightarrow t_{0} .
$$

Thus we see that $\alpha_{j-1}\left(t_{0}\right)$ is a right conditional derivative at $t_{0}$ of $\lambda_{j}-\alpha_{j}$ with respect to $\phi$, for $j \geqq 1$ and all $t_{0}$ in $(0,1)$. 
Since $\alpha_{j}=0$ for $j>m$ and since $\lambda_{j}=0$ for $j>n$, if $m>n$ we see that $\alpha_{m}(t)$, which is a right conditional derivative at $t$ of $\lambda_{m+1}-\alpha_{m+1}=0$ with respect to $\phi$, must vanish for $t$ in $(0,1)$. Thus $\alpha_{m}=0$. The argument can then be continued to show step by step that $\alpha_{i}=0$ for $i \geqq n$.

Therefore $\alpha_{n-1}(t)$ is a right conditional derivative at the point $t$ of $\lambda_{n}-\alpha_{n}=\lambda_{n}$ with respect to $\phi$, for all $t$ in $(0,1)$. If $\alpha_{n-1}(t) \neq 0$, this implies that $\left[\alpha_{n-1}(t)\right]^{-1}$ is a right conditional derivative at $t$ of $\phi$ with respect to $\lambda_{n}$. Since $\alpha_{n-1}$ is continuous on the right, we can find $u>t$ and $r>0$ such that $\left|\alpha_{n-1}(x)\right|>r$ for $x$ in $[t, u)$. Therefore for $x$ in $[t, u)$ we see that $\left|\alpha_{n-1}(x)\right|^{-1}<r^{-1}$ and $\left[\alpha_{n-1}(x)\right]^{-1}$ is a right conditional derivative at $x$ of $\phi$ with respect to $\lambda_{n}$. Hence there exist points $x^{\prime}$ arbitrarily close to $x$ on the right with $\mid\left[\phi\left(x^{\prime}\right)-\phi(x)\right]$ . $\left[\lambda_{n}\left(x^{\prime}\right)-\lambda_{n}(x)\right]^{-1} \mid<r^{-1}$. Given any $x$ and $y$ in $[t, u), x<y$, let $T$ be the set of all $x^{\prime}$ in $[x, y]$ for which there exists $x^{\prime \prime}$ in $\left[x^{\prime}, y\right]$ with $\left|\phi\left(x^{\prime \prime}\right)-\phi(x)\right| \leqq r^{-1} \int_{x}^{x^{\prime}}\left|d \lambda_{n}\right|$. Obviously $x \in T$. Also $T$ is a closed subset of $[x, y]$ because $\left|\phi\left(x^{\prime \prime}\right)-\phi(x)\right|$ is a continuous function of $x^{\prime \prime}$. To show that $T$ is open in $[x, y]$, take any $x^{\prime}$ in $T$, and choose $x^{\prime \prime}$ as above. If either $x^{\prime}=y$ or $x^{\prime}<x^{\prime \prime}$, then $\left[x, x^{\prime \prime}\right] \subset T$ is a neighborhood of $x^{\prime}$ in $[x, y]$. On the other hand, if $x^{\prime}=x^{\prime \prime}<y$, then the above considerations show that there exists $w$ in $\left(x^{\prime}, y\right]$ with

$$
\left|\phi(w)-\phi\left(x^{\prime}\right)\right|<r^{-1}\left|\lambda_{n}(w)-\lambda_{n}\left(x^{\prime}\right)\right| .
$$

Thus we have

$$
\begin{aligned}
&|\phi(w)-\phi(x)| \leqq\left|\phi(w)-\phi\left(x^{\prime}\right)\right|+\left|\phi\left(x^{\prime}\right)-\phi(x)\right| \\
&<r^{-1}\left|\lambda_{n}(w)-\lambda_{n}\left(x^{\prime}\right)\right|+r^{-1} \int_{x}^{x^{\prime}}\left|d \lambda_{n}\right| \leqq r^{-1} \int_{x}^{w}\left|d \lambda_{n}\right| .
\end{aligned}
$$

Therefore $w \in T$ so that $[x, w] \subset T$. Thus $[x, w]$ is a neighborhood of $x^{\prime}$ in $[x, y]$. Hence $T$ is both open and closed in $[x, y]$. Since $x \in T, T=[x, y]$. Therefore $y \in T$, so that $|\phi(y)-\phi(x)| \leqq r^{-1} \int_{x}^{y}\left|d \lambda_{n}\right|$ for all $x$ and $y$ in $[t, u)$. Therefore $\phi$ has bounded variation on $[t, u]$, so that $\phi[t, u]$ is a rectifiable sub-arc of $C$. This contradicts the hypothesis. This contradiction shows that $\alpha_{n-1}(t)=0$ for all $t$ in $(0,1)$, so that $\alpha_{n-1}=0$. Having proved this, we can use the same argument to show step by step that $\alpha_{n-2}=\alpha_{n-3}=\cdots=\alpha_{0}=0$. But $\alpha_{0}(t)$ $=\int_{0}^{t} d \mu_{0}(t)$. Thus $\mu_{0}$ vanishes on all subsets of $[0,1)$. Since there is inherent symmetry between the endpoints, $\mu_{0}=0$. Then

$$
0=\alpha_{1}(t)=\int_{0}^{t}[\phi(x)-\phi(t)] d \mu_{0}(x)+\int_{0}^{t} d \mu_{1}(x)=\int_{0}^{t} d \mu_{1}(x),
$$

so that $\mu_{1}=0$. Thus we show step-by-step that $\mu_{0}=\mu_{1}=\cdots=\mu_{n}=0$. This completes the proof of Theorem 1. 
There exists Jordan arcs for which condition (2) is fulfilled. For instance, a Jordan arc which has no rectifiable sub-arcs and which has a tangent at a dense set of points will do, because the existence of a tangent implies that a Lipschitz condition of order 1 is fulfilled. To see this, assume that $C$ has a tangent at $\phi\left(t_{0}\right)$. By this we mean that the parameter $t$ can be so chosen that $\phi^{\prime}\left(t_{0}\right)$ exists and is not zero. Now if $C$ does not satisfy a Lipschitz condition of order 1 at $\phi\left(t_{0}\right)$, then for each $\delta>0$ there exists $z$ with $\left|\phi\left(t_{0}\right)-z\right|<\delta$ such that $d\left(\phi\left[0, t_{0}\right], z\right)<\left|\phi\left(t_{0}\right)-z\right| / 4$ and $d\left(\phi\left[t_{0}, 1\right], z\right)<\left|\phi\left(t_{0}\right)-z\right| / 4$. Therefore, there exist $t_{1}$ in $\left[0, t_{0}\right]$ and $t_{2}$ in $\left[t_{0}, 1\right]$ with

$$
\left|\phi\left(t_{1}\right)-z\right|<\left|\phi\left(t_{0}\right)-z\right| / 4
$$

and $\left|\phi\left(t_{2}\right)-z\right|<\left|\phi\left(t_{0}\right)-z\right| / 4$, so that $\left|\phi\left(t_{1}\right)-\phi\left(t_{2}\right)\right|<\left|\phi\left(t_{0}\right)-z\right| / 2$. Also,

$$
\begin{aligned}
& \left|\phi\left(t_{0}\right)-z\right| \\
& \leqq\left|\phi\left(t_{0}\right)-\phi\left(t_{1}\right)\right|+\left|\left(t_{1}\right)-z\right|<\left|\phi\left(t_{0}\right)-\phi\left(t_{1}\right)\right|+\left|\phi\left(t_{0}\right)-z\right| / 4,
\end{aligned}
$$

so that $3\left|\phi\left(t_{0}\right)-z\right| / 4<\left|\phi\left(t_{0}\right)-\phi\left(t_{1}\right)\right|$. Thus,

$$
\left|\phi\left(t_{1}\right)-\phi\left(t_{2}\right)\right|<2\left|\phi\left(t_{0}\right)-\phi\left(t_{1}\right)\right| / 3 \text {. }
$$

It follows that

$\gamma=\left|\phi\left(t_{1}\right)-\phi\left(t_{2}\right)\right|\left|t_{1}-t_{2}\right|^{-1}\left[\left|\phi\left(t_{0}\right)-\phi\left(t_{1}\right)\right|\left|t_{0}-t_{1}\right|^{-1}\right]^{-1}<2 / 3$.

On the other hand, as $\delta \rightarrow 0$ the quantity $\gamma$ converges to $\left|\phi^{\prime}\left(t_{0}\right)\right|$ $\cdot\left|\phi^{\prime}\left(t_{0}\right)\right|^{-1}=1$. This contradiction shows that $C$ satisfies a Lipschitz condition of order 1 at $\phi\left(t_{0}\right)$. To construct a Jordan arc which has no rectifiable sub-arcs and which has a tangent at a dense set of points, let $f$ be any continuous real function on $[0,1]$ such that $f^{\prime}$ exists at a dense set $S$ of points and such that in any sub-interval the set of points where $f^{\prime}$ does not exist has positive measure. Let $\phi(t)=t+i f(t)$, so that $\phi[0,1]=C$ is a Jordan arc having a tangent at the dense set of points $\phi(S)$. Also $C$ has no rectifiable sub-arcs, because if $\phi[t, u]$ were a rectifiable sub-arc then $\phi$ would be of bounded variation on $[t, u]$, which would imply that $f^{\prime}$ would exist almost everywhere in $[t, u]$, contrary to the condition on $f$.

It only remains to construct the function $f$. The standard techniques for the construction of nondifferentiable functions can be used (see Hobson [2]). Let $\left\{r_{n}\right\}=s$ be a sequence of irrational numbers which is dense in $[0,1]$. It is easy to construct inductively a sequence $\left\{C_{n}\right\}$ of countable subsets of $[0,1]$, each consisting of rational numbers and each containing 0 and 1 , such that the accumulation points of $C_{n}$ are exactly $r_{1}, \cdots, r_{n}$, such that the distance 
of two consecutive points $t_{1}$ and $t_{2}$ of $C_{n}$ is not larger than $6^{-n} d$, where $d$ is the distance between the sets $\left\{t_{1}, t_{2}\right\}$ and $\left\{r_{1}, \cdots, r_{n}\right\}$, and such that $D_{n} \subset C_{n+1}$. Here $D_{n}=C_{n} \cup \tilde{C}_{n}$, where $\tilde{C}_{n}$ consists of all points which lie midway between two consecutive points of $C_{n}$. Thus $D_{n}$ consists of rational numbers, and therefore is disjoint from the sequence $s$. Define the function $f_{n}$ on $[0,1]$ as follows. Let $f_{n}(t)=0$ if $t \in C_{n} \cup\left\{r_{1}, \cdots, r_{n}\right\}$. For consecutive points $t_{1}$ and $t_{2}$ in $C_{n}$, let $f_{n}(t)=3^{n}\left(t-t_{1}\right)$ if $t_{1} \leqq t \leqq\left(t_{1}+t_{2}\right) / 2$ and $f_{n}(t)=3^{n}\left(t_{2}-t\right)$ if $\left(t_{1}+t_{2}\right) / 2 \leqq t$ $\leqq t_{2}$. Since any point of $[0,1]$ which is not in $C_{n} \cup\left\{r_{1}, \cdots, r_{n}\right\}$ lies between two consecutive points of $C_{n}$, this defines $f_{n}$ uniquely at all points of $[0,1]$. The function $f_{n}$ is clearly continuous, except possibly at the points $r_{1}, \cdots, r_{n}$, and $\left|f_{n}^{\prime}(t)\right|$ exists and equals $3^{n}$ if $t$ is not in the set $D_{n} \cup\left\{r_{1}, \cdots, r_{n}\right\}$. Also, if $u_{1}$ and $u_{2}$ are consecutive points of $D_{n}$, it is clear that $\left|f_{n}\left(u_{2}\right)-f_{n}\left(u_{1}\right)\right|\left(u_{2}-u_{1}\right)^{-1}=3^{n}$. To investigate the behavior of $f_{n}$ at the point $r_{i}$, where $i \leqq n$, let $t$ be any point of $[0,1]-\left\{r_{1}, \cdots, r_{n}\right\}$. There exist consecutive points $t_{1}$ and $t_{2}$ of $C_{n}$ with $t_{1} \leqq t \leqq t_{2}$. By the definition of $f_{n}$, we have $\left|f_{n}(t)\right| \leqq 3^{n}\left(t_{2}-t_{1}\right)$. By the construction of $C_{n}$, we have $t_{2}-t_{1} \leqq 6^{-n}\left|t-r_{i}\right|^{2}$. Thus, $\mid f_{n}(t)$ $-f_{n}\left(r_{i}\right)|=| f_{n}(t)\left|\leqq 2^{-n}\right| t-\left.r_{i}\right|^{2}$ for all $t$ in $[0,1]$. In particular, we see that $f_{n}^{\prime}\left(r_{i}\right)=0$, so that $f_{n}^{\prime}(t)$ exists if $t$ is not in $D_{n}$, and that $f_{n}$ is continuous at $r_{i}$. Therefore, $f_{n}$ is continuous on $[0,1]$. We also see that $0 \leqq f_{n}(t) \leqq 2^{-n}$ for all $t$. The series $\sum_{n=1}^{\infty} f_{n}$ therefore converges uniformly on $[0,1]$ to a continuous function $f$. It will be shown that $f^{\prime}(t)$ exists if and only if $t$ is a member of the sequence $s$. If $t=r_{i}$ is a member of $s$, let $f=g+h$, where $g=\sum_{n=1}^{i-1} f_{n}$ and $h=\sum_{n=i}^{\infty} f_{n}$. Then $g^{\prime}\left(r_{i}\right)$ exists because $r_{i}$ is not in $D_{n}$ for all $n$. Also,

$$
\left|h(u)-h\left(r_{i}\right)\right| \leqq \sum_{n=i}^{\infty}\left|f_{n}(u)-f_{n}\left(r_{i}\right)\right| \leqq \sum_{n=i}^{\infty} 2^{-n}\left|u-r_{i}\right|^{2} \leqq\left|u-r_{i}\right|^{2}
$$

for all $u$, so that $h^{\prime}\left(r_{i}\right)$ exists and is zero. Therefore $f^{\prime}\left(r_{i}\right)$ exists. Now assume that $t$ is not an element of the sequence $s$. Let $n$ be arbitrary. Since $t$ is not an accumulation point of $C_{n}$, there exist consecutive points $u_{1}$ and $u_{2}$ in $D_{n}$ with $u_{1} \leqq t \leqq u_{2}$. Then $f\left(u_{2}\right)-f\left(u_{1}\right)=\sum_{k=1}^{n} f_{k}\left(u_{2}\right)$ $-f_{k}\left(u_{1}\right)$ because $f_{k}(u)=0$ if $u \in D_{n} \subset C_{n+1}$ and $k \geqq n+1$. Thus

$$
\begin{aligned}
& \left|f\left(u_{2}\right)-f\left(u_{1}\right)\right|\left(u_{2}-u_{1}\right)^{-1} \\
& \quad \geqq\left|f_{n}\left(u_{2}\right)-f_{n}\left(u_{1}\right)\right|\left(u_{2}-u_{1}\right)^{-1}-\sum_{k-1}^{n-1}\left|f_{k}\left(u_{2}\right)-f_{k}\left(u_{1}\right)\right|\left(u_{2}-u_{1}\right)^{-1} \\
& \quad \geqq 3^{n}-\sum_{k=1}^{n} 3^{k} \geqq n .
\end{aligned}
$$

Thus the difference quotients, $\left[f\left(u_{2}\right)-f\left(u_{1}\right)\right]\left(u_{2}-u_{1}\right)^{-1}$, for $u_{1} \leqq t \leqq u_{2}$, 
are not bounded. It follows that $f^{\prime}(t)$ does not exist, as was to be proved.

\section{REFERENCES}

1. S. Banach, Théorie des opérations linéaires, Warsaw, 1932.

2. E. W. Hobson, The theory of functions of a real variable, vol. 2, Cambridge, 1926.

3. S. N. Mergelyan, Uniform approximations to functions of a complex variable, Amer. Math. Soc. Translation no. 101, Providence, 1954.

University of California, Berkeley

\section{AN IDENTITY IN THE THEORY OF THE GENERALIZED POLYNOMIALS OF JACOBI}

\section{B. GERMANSKY}

1. Introduction of some new notations in the theory of the Jacobi polynomials. To facilitate the passage from the usual Jacobi polynomials $P_{n}^{(\alpha, \beta)}(x)$ to the generalized Jacobi polynomials $P_{n}^{\left(\alpha_{0}, \ldots, \alpha_{p}\right)}(x)$ considered here, we introduce some new notations in the theory of the first mentioned polynomials. It is well known ${ }^{1}$ that the zeros of these polynomials are the points $x_{1}=x_{1}^{(n)}, x_{2}=x_{2}^{(n)}, \cdots, x_{n}=x_{n}^{(n)}$, which maximize the expression

$$
T\left(x_{1}, x_{2}, \cdots, x_{n}\right)=T(x)=\prod_{k=1}^{n}\left(1-x_{k}\right)^{p}\left(1+x_{k}\right)^{q} \prod_{1 \leqq \nu<\mu \leqq n}\left|x_{\nu}-x_{\mu}\right|
$$

in the unit-interval $I:[-1,+1]$. Here $\alpha=2 p-1$ and $\beta=2 q-1$ and it is assumed $x_{1}>x_{2}>\cdots>x_{n}$. Instead of $T(x)$ we use the expression

$$
V_{m}\left(\xi_{1}, \xi_{2}, \cdots, \xi_{m} ; e_{1}, e_{2}, \cdots, e_{m}\right)=V_{m}(\xi ; e)=\prod_{1 \leqq i<k \$ m}\left(\xi_{i}-\xi_{k}\right)^{e_{i e k}},
$$

where we suppose that $m=n+2$; that the points $\xi_{1}$ and $\xi_{m}$ are fixed from the outset and are equal to $a_{0}=-1$ and $a_{1}=+1$ respectively; that $e_{1}=\rho_{0}=q, e_{m}=\rho_{1}=p, e_{2}=e_{3}=\cdots=e_{m-1}=1$; that the points $\xi_{1}, \xi_{2}, \cdots, \xi_{m}$ are counted in increasing order; $-1=\xi_{1}<\xi_{2}<\ldots$ $<\xi_{m-1}<\xi_{m}=+1$ and therefore that $\xi_{2}=x_{n}, \xi_{3}=x_{n-1}, \cdots, \xi_{m-1}=x_{1}$. It results that $V_{m}\left(\xi_{1}, \xi_{2}, \cdots, \xi_{m} ; e_{1}, e_{2}, \cdots, e_{m}\right)$ is a function of $\xi_{2}, \xi_{3}, \cdots, \xi_{m-1} ; \rho_{0}, \rho_{1}$ only, as is $T(x)$. Then the zeros of the Jacobi polynomial $P_{n}^{(\alpha, \beta)}(x)$ are the points $\xi_{2}=\xi_{2}^{(m)}=x_{n}^{(n)}, \xi_{3}=\xi_{3}^{(m)}=x_{n-1}^{(n)}, \cdots$, $\xi_{m-1}=\xi_{m-1}^{(m)}=x_{1}^{(n)}$, which maximize the absolute value of $V_{m}(\xi ; e)$ on $I$, under the mentioned conditions. We call the last function the generalized Vandermondean of the degree $m$ and of the order 1 . We write

Received by the editors January 2, 1958 and, in revised form, April 25, 1958.

${ }^{1}$ Szegö, Orthogonal polynomials, New York, 1939, p. 136, Theorem 6.7.1. 